\title{
Reply to Chien et al
}

To the Editor-We are thankful for the comments made by Chien et al, which enrich the discussion on the important issue of vascular complications caused by nontyphoid Salmonella (NTS) isolates [1]. We do agree that it is a limitation of the present study addressed by Chien et al that only $42.2 \%$ of the patients underwent imaging studies to exclude the presence of vascular infections [2]. As mentioned, we had recognized that the prevalence of vascular infections was likely to be underestimated, especially among those without imaging studies. However, the prediction capabilities of the scores derived from the selected 151 patients and that of the initial study population were comparable (the area under the receiver operating 
characteristic curve was 0.80 and 0.83 , respectively). The increment of the number of cases with imaging studies may increase the predictive power and applicability of the clinical score.

Chien et al also raised the issue of symptom duration before medical care [1]. Our previous studies showed that vascular infections were less frequently found in patients with malignancy and connective diseases if they were affected by NTS bacteremia [3, 4], and such a finding was supported by other reports in Taiwan [5]. A multicenter prospective study enrolling adults with NTS bacteremia to undergo computed tomography (CT) scanning is ongoing. The study will include more potential risk factors, such as the duration of symptoms and the severity of arterial atherosclerosis, into consideration. In addition, with clinical experience of an infected aortic aneurysm developing 3 months later even with 3 weeks of antimicrobial therapy [6], 6 months of follow-up is considered appropriate to exclude the likelihood of infected aortitis after the onset of NTS bacteremia.

Clinical courses of patients with NTS bacteremia may vary greatly. The decision of undergoing CT or magnetic resonance imaging (MRI) to screen the vascular infection should be individualized. Our results could help clinicians to recognize a high-risk population of infected vascular lesions among adults with NTS bacteremia, and justify comprehensive radiological surveys, but did not argue against the necessity of clinical alertness for vascular infections among immunosuppressed individuals aged $>50$ with NTS bacteremia, for whom it seems to be unreasonable to recommend imaging studies due to economic concerns and contrast medium-related adverse effects. In our study cohort, if the NTSVI score were $<0$ (ie, -1 or -2 ), all 43 patients with imaging studies had no vascular infections. This negative finding would certainly question the need of imaging surveys to exclude the existence of vascular lesions. Moreover, it was noteworthy that half of the patients with a risk of vascular infection were not surveyed by CT or MRI scans in our study population. Therefore, it is prudent to recommend that the patients identified as being at a risk of vascular infections by the scoring algorithm need further imaging studies.

\section{Note}

Potential conflicts of interest. All authors: No reported conflicts.

All authors have submitted the ICMJE Form for Disclosure of Potential Conflicts of Interest. Conflicts that the editors consider relevant to the content of the manuscript have been disclosed.

Po-Lin Chen, ${ }^{1,3,4}$ Hung-Jen Tang, ${ }^{5,6}$ and Wen-Chien $\mathrm{Ko}^{1,2,3}$

${ }^{1}$ Department of Internal Medicine, and ${ }^{2}$ Center for Infection Control, National Cheng Kung University Hospital; ${ }^{3}$ Department of Medicine, and ${ }^{4}$ Graduate Institute of Clinical Medicine, College of Medicine, National Cheng Kung University; ${ }^{5}$ Department of Medicine, Chi Mei Medical Center; and ${ }^{6}$ Department of Health and Nutrition, Chia Nan University of Pharmacy and Science, Tainan, Taiwan

\section{References}

1. Chien KS, Farmakiotis D, Rodriguez-Barradas MC, Musher DM. It's not that simple. Clin Infect Dis 2012; 56:308.

2. Chen PL, Lee CC, Li CY, et al. A simple scoring algorithm predicting vascular infections in adults with nontyphoid Salmonella bacteremia. Clin Infect Dis 2012; 55:194-200.

3. Huang CF, Chen PL, Liu MF, et al. Nontyphoidal Salmonella bacteremia in patients with connective tissue diseases. J Microbiol Immunol Infect 2012; 45:350-5.

4. Li CW, Chen PL, Lee NY, et al. Non-typhoidal Salmonella bacteremia among adults: an adverse prognosis in patients with malignancy. J Microbiol Immunol Infect 2012; 45: 343-9.

5. Hsu RB, Tsay YG, Chen RJ, Chu SH. Risk factors for primary bacteremia and endovascular infection in patients without acquired immunodeficiency syndrome who have nontyphoid salmonellosis. Clin Infect Dis 2003; 36:829-34

6. Chen PL, Tsai LM, Kan CD, Ko WC. Is 2 weeks of antibiotic therapy enough to treat elderly patients with nontyphoid Salmonella bacteremia? A case report of fatal endovascular infection. J Microbiol Immunol Infect. doi:10.1016/j.jmii.2012.03.008.
Correspondence: Wen-Chien Ko, MD, Division of Infectious Diseases, Department of Internal Medicine, National Cheng Kung University Hospital, No. 138, Sheng Li Road, Tainan 704, Taiwan (winston3415@gmail.com).

Clinical Infectious Diseases 2013;56(2):308-9

(C) The Author 2012. Published by Oxford University Press on behalf of the Infectious Diseases Society of America. All rights reserved. For Permissions, please e-mail: journals. permissions@oup.com.

DOI: 10.1093/cid/cis835 